\title{
On the Multiple Event Detection in Atom Probe Tomography
}

\author{
Zirong Peng ${ }^{1}$, Baptiste Gault ${ }^{1}$, Dierk Raabe ${ }^{1}$, Michael W Ashton ${ }^{2}$, Susan B Sinnott ${ }^{3}$, Pyuck-Pa Choi ${ }^{1,4}$ \\ and Yujiao $\mathrm{Li}^{5}$ \\ 1. Department of Microstructure Physics and Alloy Design, Max-Planck-Institut für Eisenforschung \\ GmbH, Max-Planck-Straße 1, Düsseldorf, Germany \\ 2. Department of Materials science and Engineering, University of Florida, Gainesville, Florida USA \\ 3. Materials Science and Engineering, Pennsylvania State University, State College, Pennsylvania, USA \\ 4. Department of Materials Science and Engineering, Korea Advanced Institute of Science and \\ Technology (KAIST), 291 Daehak-ro, Yuseong-gu, Daejeon, Republic of Korea \\ 5. Center for Interface-Dominated High Performance Materials, Ruhr-Universität Bochum, Bochum, \\ Germany
}

Atom probe tomography (APT) is a state-of-art advanced microanalytical technique, capable of threedimensional elemental mapping at the atomic scale. It is a combination of ion projection microscopy and time-of-flight (TOF) mass spectrometry. During APT analysis, a high positive voltage (a few $\mathrm{kV}$ ) is applied to the sharp, needle-sharped specimen (radius of curvature $<200 \mathrm{~nm}$ ) to create a strong standing electric field (in the range of $10^{10} \mathrm{Vm}^{-1}$ ). In addition to this base field, high-voltage (HV) pulses or laser pulses are used to trigger and time-control the field evaporation of the surface atoms of the specimen, a process where they are emitted as ions. These positively charged ions are then accelerated and projected onto a detector. Upon hitting the detector, their arrival sequences (corresponding to the $\mathrm{Z}$ coordinate), impact positions (corresponding to the $\mathrm{X}$ and $\mathrm{Y}$ coordinates) and TOFs (corresponding to the elemental identity) are recorded, which are used to reconstruct the three dimensional elemental map [1]. Therefore, the accuracy of the measurement largely depends on the capability of the detector to properly resolve and detect the hit events.

Field evaporation is a probabilistic process. All the surface atoms have a certain probability to field evaporate. Hence, the emission of a pulse can induce blank events (no ion is evaporated), single events (only one ion is evaporated) or multiple events (more than one ion are evaporated). Besides, correlated evaporation, where the departure of one atom leads to the successive evaporation of its close neighbors at the surface, has previously been reported [2], which also contributes to the amount of multiple events. In some extreme cases, 'catastrophic' evaporation where a burst of ions are evaporated nearly simultaneously may also take place [3]. Furthermore, dissociation of metastable molecular ions under the influence of the strong electrical field is also known to give rise to multiple events [4].

Current detectors utilized in the commercial APT instruments are delay-line detectors (DLD), consisting of a stack of microchannel plates (MCP) and three delay-lines (DL) [5]. It is a proven technique with a very good performance on the detection of single and multiples events. However, like most DLDs, the existence of the electronic 'dead time' and 'dead zone' after each hit will limit their multiple event detectability. If the constituent ions of the multiple event are too closely correlated in time and space, i.e. the intervals between their arrival time are shorter than the 'dead time' and the distances between their impact positions are within the 'dead zone', some signals will be lost and the resulting 3D elemental information will be biased $[6,7]$.

In this work, we use cemented tungsten carbide because it is a well-known issue that APT analysis of 
carbides suffered from the above mentioned problem [8], which is normally referred to as ion pile-up or detector saturation effect. Two cutting-edge atom probe microscopes, namely LEAP ${ }^{\mathrm{TM}} 5000 \mathrm{XS}$ and LEAP $^{\mathrm{TM}} 5000$ XR (CAMECA Instruments, Madison, WI, USA) are applied. Detailed analysis of multiple events were done with the implantation of DFT (density-functional theory) calculation. Information about the detector dead time and dead zone are extracted and the seriousness of the ion pile-up effect are discussed on the basis of the intrinsic character of the multiple events. Figure 1 shows the spatial distance between the successive ions detected in multiple events as a function of their TOF differences, which highlights the non-trivial nature of the potential losses of signals on a CAMECA LEAP 5000 XS device. We can see that the behaviors of carbon and tungsten are different, which could explain some speciesspecific losses that result in compositional biases.

\section{References:}

[1] B. Gault et al, Atom Probe Microscopy, (Springer, New York) 2012.

[2] F. De Geuser et al, Surf. Sci. 601 (2007) p. 536.

[3] W. Lefebvre et al, Surf. Interface Anal. 39 (2007) p. 206.

[4] B. Gault et al, New J. Phys. 18 (2016) p. 33031.

[5] G. Da Costa et al, Rev. Sci. Instrum. 83 (2012) p. 123709.

[6] O. Jagutzki et al, IEEE Trans. Nucl. Sci. 49 (2002) p. 2477.

[7] F. Meisenkothen et al, Ultramicroscopy. 159 (2015) p. 101.

[8] Z. Peng et al, Microsc. Microanal. (2017) p. 1.

(a)

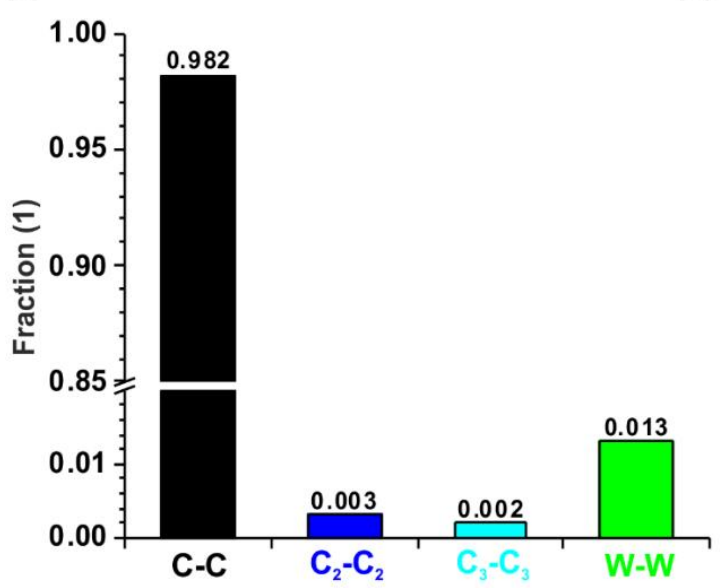

(b)

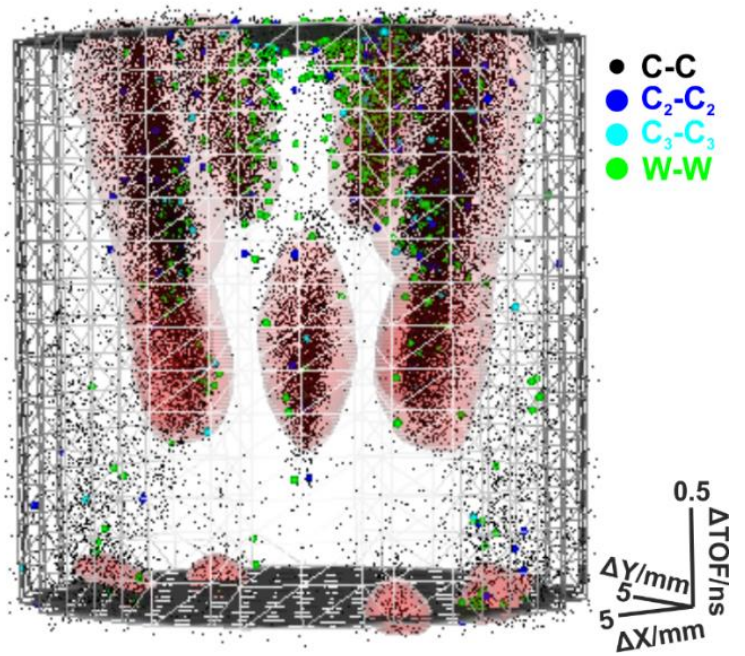

Figure 1. (a) Fractions of different ion pairs detected in the multiple events. Here, only the pairs consisting of the same type of ions are included because they are the only ones suffering from the detector pile-up effect and may not be properly recorded by the detector. That the amount of the carbon ion pairs is much higher than the tungsten ion pairs is the essential reason why the resulting chemical composition is biased. (b) 3D map showing the distribution of TOF and impact position differences between the two constituent ions of all the ion pairs listed in (a). Each dot represents one ion pair. The high density areas, marked in red, are the domains in time and space where second ion in the pair has been detected. While, the low density areas with very limited amount of ion pairs represent the status when the detector did not work properly, i.e. they are regions of the detector dead time and dead zone. 